\title{
Simulation of multi-layer GEM from single to triple GEMs
}

\author{
Aera JUNG, ${ }^{a, *}$ Yong BAN, ${ }^{a}$ Dayong WANG, ${ }^{a}$ Yue WANG ${ }^{a}$ and Licheng ZHANG ${ }^{a}$ \\ ${ }^{a}$ School of Physics, Peking University, \\ 209 Chengfu Rd, Haidian District, Beijing, China \\ E-mail: aera@pku.edu.cn, bany@pku.edu.cn, dayong.wang@pku.edu.cn, \\ 1801210129@pku.edu.cn, zhanglic@pku.edu.cn
}

Originally Micro-Pattern Gas Detectors (MPGDs), a type of gaseous ionization detector, were developed for high energy physics experiments, however applications have expanded to astrophysics, neutrino physics, neutron detection, and medical imaging. Over the past 20 years this led to the development of novel MPGD devices: the Micro-Strip Gas Chamber (MSGC), Gas Electron Multiplier (GEM), Micromegas (a type of MPGD using a parallel-plate micro-mesh avalanche counter), and many others, revolutionizing cell size limits for many gas detector applications and considerably improving reliability and radiation hardness.

In a gaseous detector, a particle (or an energetic photon) enters a gas cell and collides with an atom of gas, which emits a high energy electron. This electron undergoes further collisions which creates an ionization tract whose electrons are drifted by a small electric potential across a gas cell onto a bottom plate consisting of a double layered conductor separated by an insulator with a strong electric field $(50-70 \mathrm{kV} / \mathrm{cm})$ difference between them. This bottom plate, called a Gas Electron Multiplier (GEM), has an array of tiny holes and the ionization tract electrons fortunate enough to pass though the holes are strongly accelerated causing them to create secondary cascades in the direction of a pixel readout array such as the complementary metal-oxide-semiconductor application-specific integrated circuit (CMOS ASIC) chip.

With a multi-GEM layer structure, of up to 5 layers, a very high effective gain (up to $10^{6}$ with some gases) can be attained with each GEM layer working at an individually much lower gain thus avoiding discharge problems. This is the major advantage of the GEM technology. In many groups, triple GEM (3 layers of GEMs) designs have been extensively studied in both experiments and simulations.

Here, we will present a simulation study of single, double, and triple GEMs's to characterize their properties such as gain, spatial resolution, energy resolution, efficiency, and so on using Garfield++ and ANSYS field solver and compare between the results of published experiments and simulations.

$37^{\text {th }}$ International Cosmic Ray Conference (ICRC 2021)

July 12th - 23rd, 2021

Online - Berlin, Germany

\footnotetext{
${ }^{*}$ Presenter
} 


\section{Introduction}

Originally Micro-Pattern Gas Detectors (MPGDs) [1], a type of gaseous ionization detector, were developed for high-energy physics experiments. Their applications have expanded to astrophysics, neutrino physics, neutron detection, and medical imaging. Most advances in high-energy, nuclear, and astrophysics have been facilitated by the continuing pursuit of increasingly capable detectors. The past 20 years has led to the development of novel MPGD devices: the Micro-Strip Gas Chamber (MSGC) [2], Gas Electron Multiplier (GEM) [3], Micromegas (a type of MPGD using a parallel-plate micro-mesh avalanche counter) [4], and many others, while revolutionizing cell size limits for many gas detector applications and considerably improving reliability and radiation hardness. The MPGD structures can be grouped into two large families, micromech-based detectors and hole-type structures. Micromesh-based structures include: Micromegas, "Bulk" Micromegas, "Microbulk" Micromegas and "InGrid." The hole-type structures are: GEMs, thickGEMs (THGEM), patterned resistive thick GEM devices (RETGEM) and "Micro-Hole and Strip Plate" (MHSP) elements [5].

In 1997 Fabio Sauli introduced GEM technology (a type of MPGD using a hole-type structure) [3], development was pursued by several groups, and GEMs were successfully used in many experiments. A GEM electrode is a polymer thin-foil, copper-clad on both sides, with a high density of holes. On application of a high voltage gradient between the two sides of the copper cladding, and in an appropriate gas environment, electrons released by ionization on one side of the electrode drift into the holes and multiply. GEMs have a number of advantages that make them very attractive for various applications including flexible geometry, adjustable size, good spatial resolution, low manufacturing cost, and computationally undemanding track reconstruction.

These advantages are a considerable advance over competing systems and GEMs have been used successfully in a wide variety of experiments. However, the development of modern detectors, including GEM-based detectors, presents formidable challenges, which are of great technological, engineering, and organizational complexity. Development time-scales have stretched over multiple decades starting from the concept, progressing through intensive $R \& D$, design and prototyping, mass production, installation, and finally system integration and commissioning. It is important to understand the existing detector technologies and the concept \& design of future advanced detectors. Also, optimum operation of GEM detectors necessitates careful and detailed study of various detector aspects. The parameters of the GEM foil, overall geometry of the detector, the gas mixture to be used, and so on, are determined by the experimental requirements for tracking and timing precision. While the ultimate choice of design parameters can be finalized only after successful experimental demonstration, a suitable numerical simulation tool can aid the design decision process significantly.

Therefore, this proceeding paper is an effort to run GEM detector simulations to match published experimental results [6] for different designs of gas detectors. Usually, the Garfield++ simulation toolkit [7] is used for gaseous detector simulations including those using GEMs. This simulation toolkit incorporates Heed [8] for estimating primary ionization and MAGBOLTZ [9] to analyze the effects of using a gas mixture consisting of $70 \%$ argon and $30 \%$ carbon dioxide. The resulting simulation is extremely capable and is used by a very large community of scientists. Through these simulations, we are going to understand and optimize the detector in depth and 
present the direction for the next generation of detectors.

\section{Gas Electron Multiplier (GEM)}

The Gas Electron Multiplier (GEM) detector consists of a thin-foil deposited on each side of an insulator to construct a copper-insulator-copper sandwich which is perforated obtaining a high density of holes. By applying a significant voltage difference between the two copper foil layers a strong electric field is created within the holes, causing electron avalanches to multiply incident electrons. Physically, the central insulator is usually (in the original design) a thin film of the polymer Kapton, typically with a thickness of $50 \mu \mathrm{m}$ [3]. An array of holes, with typical diameter of $50-70$ $\mu m$ and a $100-150 \mu m$ spacing, is photo-lithographically etched into this foil. Via application of a potential difference of several hundred volts between the two sides of the copper electrodes, a strong electric field is generated inside these holes. Each hole acts as an independent proportional counter. Electrons released by a primary ionization particle in the upper conversion region (above the GEM foil) drift into the holes, where charge multiplication occurs in the high electric field $(50-70 \mathrm{kV} / \mathrm{cm})$. Most of the avalanche electrons are transferred into the gap below the GEM. Several GEM foils can be stacked together, allowing multi-layer GEM detectors to operate at an overall gain above $10^{4}$ in the presence of highly ionizing particles while strongly reducing the risk of discharges. With such a multi-GEM layer (of up to 5 layers) structure, a very high effective gain can be attained with each GEM layer working at an individually much lower gain and electric potential thus avoiding discharge problems. This is a major advantage of the GEM technology. Localization can then be performed by collecting the charge on a patterned one or two-dimensional readout board, with an arbitrary pattern, placed below the last GEM. The use of such a two-dimensional readout with a gas-based detector is known as a Gas Pixel Detector (GPD) as it generates a pixelated image output [10]. The performance and robustness of these GEM based detectors has encouraged their use in high-energy and nuclear physics, UV and visible photon detection, astroparticle and neutrino physics, neutron detection, and medical physics. Most structures were originally optimized for high-rate particle tracking in nuclear and high-energy physics experiments. Due to their many advantages (ease of manufacturing since large-area GEMs can in principle be mass-produced, flexible geometry for the GEM and readout pads, and so on) GEMs are being used, researched, and developed for many experiments.

\section{GEM simulation model}

This simulation is based on Garfield++ which is a C++ version of Garfield [11]. Garfield and Garfield++ are a computer simulation packages developed by CERN for modeling particle detectors with gas particle interactions such as drift chambers. Garfield is the original version written in Fortran and Garfield++ is a newer version using C++. Of course, Garfield++ shares functionality with Garfield and is built on the ROOT platform (a data analysis program developed by CERN) [12] with allows the user to easily view the drift lines of electrons and ions, and also to do basic filtration of data by adding user-made functions. Unfortunately, Garfield++ can not compute complex electric fields, therefore the commercial software ANSYS is separately applied to the GEM detector geometry to create a model of the detector's electric field. For our GEM geometry, we 


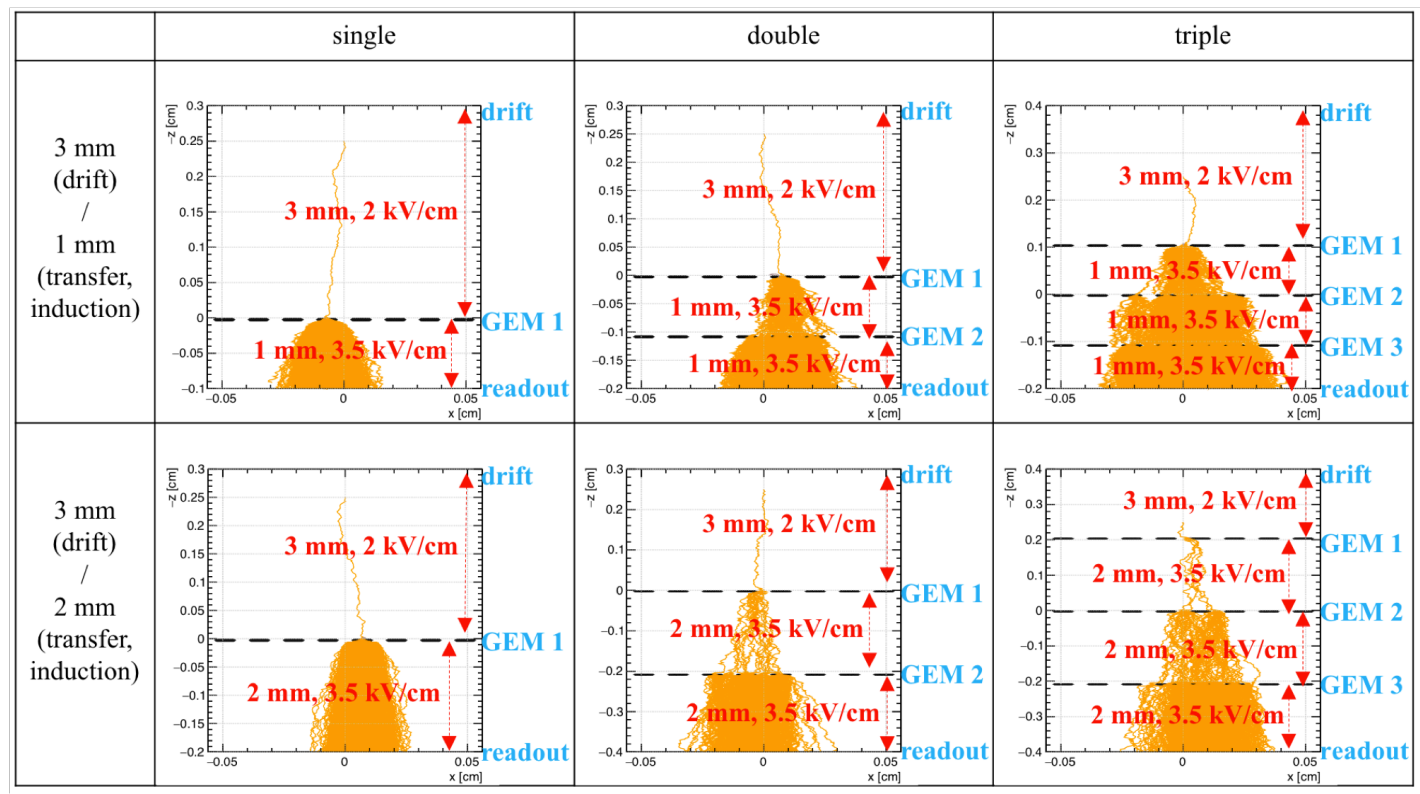

Figure 1: Simulation of electron avalanche trajectories in a single, double, and triple GEMs. The electric field configuration is characterized by the drift field in the drift gap, the transfer field in the transfer gap, the induction field in the induction gap, and the voltage difference $\triangle$ GEM V between the top and bottom GEM voltage (i.e. the conductive layers on each side of the Kapton insulator).

considered a standard hexagonal GEM foil with a pitch of $140 \mu \mathrm{m}$, a outer hole diameter of $70 \mu \mathrm{m}$, an inner hole diameter of $50 \mu m$, and a Kapton thickness of $50 \mu m$ with $5 \mu m$ copper conductive layers on both sides, and filled with a gas mixture of $70 \%$ argon and $30 \%$ carbon dioxide.

We have been simulating and studying single, double, and triple GEM configurations using this GEM model. On our multi-GEM simulations, voltages across each GEM were kept identical, with transfer and induction fields of $3.5 \mathrm{kV} / \mathrm{cm}$ and drift fields of $2 \mathrm{kV} / \mathrm{cm}$ as shown in Fig. 1. The drift distance is kept at a consistent $3 \mathrm{~mm}$, however transfer (spacing between the different GEM foils) and induction distances (spacing between last GEM foil and readout) of both 1 and $2 \mathrm{~mm}$ are used.

Our simulation results will give us a better understanding of how electrons are transported by various detector field configurations and therefore allow us to operate the detector in a more efficient way. In other words, after general simulation results for single, double, and triple GEM systems, the several characteristics of a GEM detector can be investigated through simulations using ANSYS and Garfield++. From these general considerations, we propose to improve and better understand our GEM based detector characteristics.

\section{Results}

We simulated the GEM detector using the parameters mentioned above and focused on determining the gain, electron transparency, efficiency, spatial resolution, and energy resolution by varying the electron flow into single layer, double layer, and triple layer GEM systems. For these 


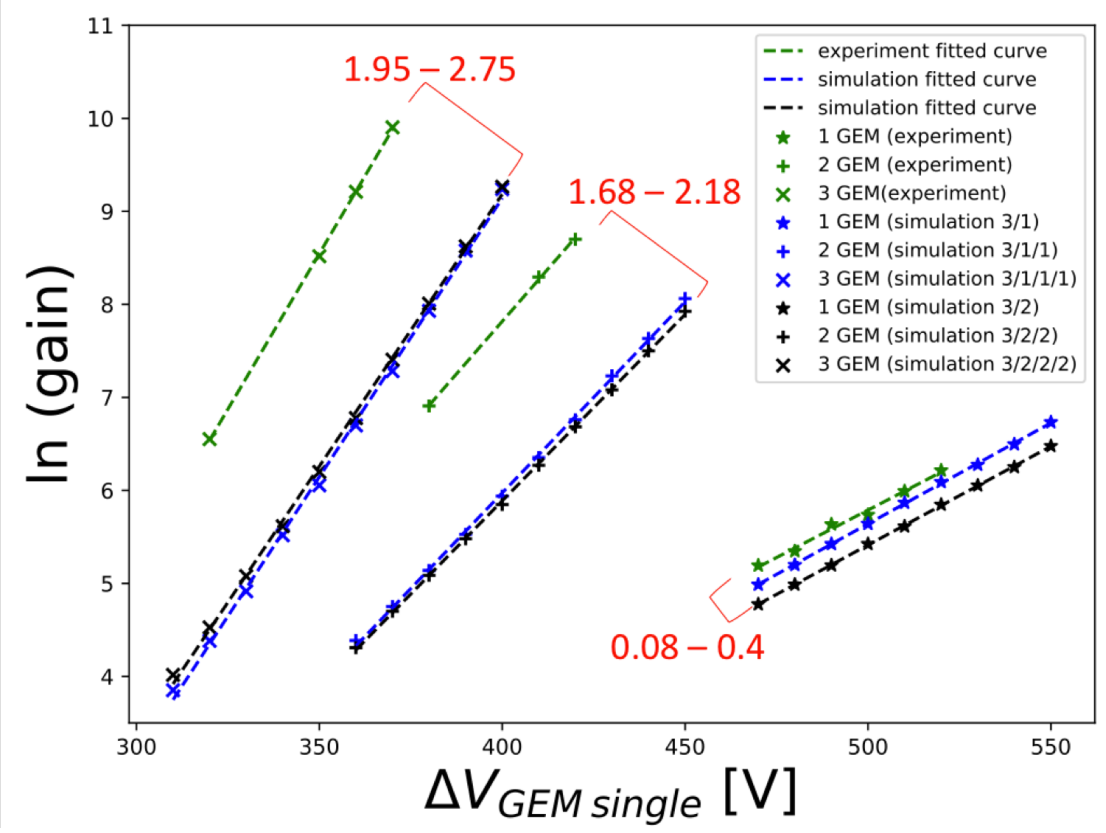

Figure 2: Green is the experimental data (originally from Bachmann's paper [6] by eye), blue is the simulation model with a transfer and induction gap of $1 \mathrm{~mm}$, and black is the simulation model with a transfer and induction gap of $2 \mathrm{~mm}$. Moreover, the star symbol is a single GEM, the cross symbol is a double GEM, and the " $x$ " symbol is a triple GEM. As the number of layers of GEM increases, the difference between experimental and simulation results increases.

simulations, the penning transfer ratio is manually set to 0.57 as given in [13] and the gas is set to $1 \mathrm{~atm}$ pressure at room temperature. For initiating the avalanche process, a single electron with 0.5 $\mathrm{eV}$ energy is placed $2.995 \mathrm{~mm}$ above the top of the first GEM.

\subsection{Gain}

The avalanche process is governed by probabilities and therefore the final size of an avalanche started by a single electron fluctuates. That is, the gain (i.e. the multiplication of a incoming electron signal) in a gaseous multiplier, is achieved via the creation of new electron-ion pairs. A new electron-ion pair is created when the energy transferred from a collision is greater than the ionization energy of the gas atom or molecule. Once the electron has gained enough energy, due to acceleration in the electric field, a new electron-ion pair can be created. This process creates an avalanche of electrons. That is, gain is given by the number of electrons created by each primary electron that reaches the anode.

Gain varies with almost every parameter involved in the operation of a GEM. In this paper, these include GEM voltage and the number of GEM layers (i.e. single, double, or triple GEMs). Here we have performed a number of simulations in order to identify how to optimize these parameters for detector operation.

This is very well known issue in which the gain seen in experiments exceeds that which can the obtained in the simulations results. Many groups are doing research on this topic, but we 

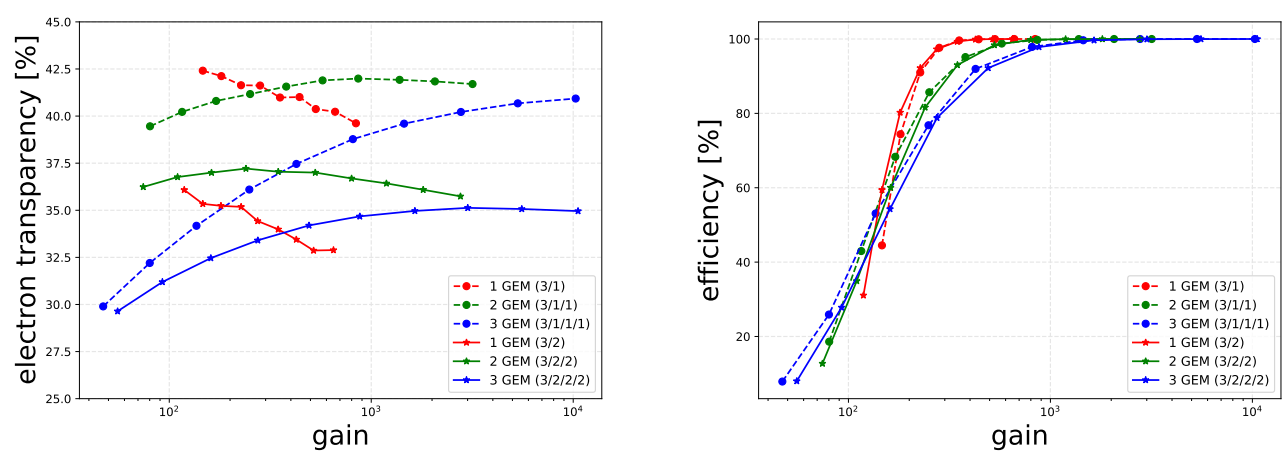

Figure 3: Left: Total electron transparency as a function of gain for single (red), double (green), and triple (blue) GEMs. Right: Efficiency as a function of gain for single (red), double (green), and triple (blue) GEMs.

still do not understand exactly why this discrepancy exists. So far, simulations cannot achieve the nano, let alone sub-nano, scale resolution required to accurately reflect the short times in which the microscopic interaction phenomena to occur. Therefore the gain seen in simulations is lower. We compared published experimental data [6] with our simulations. As shown in Fig.2, the difference between simulation and experimental data for a single GEM detector is about $0.08-0.4$, double GEM is about $1.68-2.18$, and triple GEM is about $1.95-2.75$. As the number of GEM layers increases, the difference between experimental and simulation results increases.

\subsection{Electron transparency}

The electric field inside the GEM holes defines the intrinsic gain properties while the electric field outside the holes and the electron diffusion effect determine the GEM's transparency. When electric field line stops on the GEM then the transparency is reduced. Some of these lines above the GEM do not enter the GEM holes and some others coming from the holes stop below the GEM. This influences the gain of the GEM because a certain fraction of the electrons stops on the GEM foil. In Bonivento's paper [14], the dependency of the transparency on the two electric fields outside the GEM foil is shown. In this paper, however we compare the total transparency of single, double, and triple GEMs. There are some differences, but overall the total electron transparency of single, double, and triple GEMs is about 35\%, as shown in the left panel of Fig. 3. In addition, the larger the induction and transfer gaps, the lower the total electron transparency value.

\subsection{Efficiency}

The efficiency describes the probability of a trespassing particle to yield the expected signal and, if applicable, to overcome a threshold value needed have this signal recognized. We used the same threshold for single, double, and triple GEMs. The efficiency increases with gain and then comes to a plateau after a certain gain value is reached, as shown in the right panel of Fig. 3. It is to be noted that the efficiency plateau starts from a similar gain value (around gain 1000) in all single, double, and triple GEM systems. 

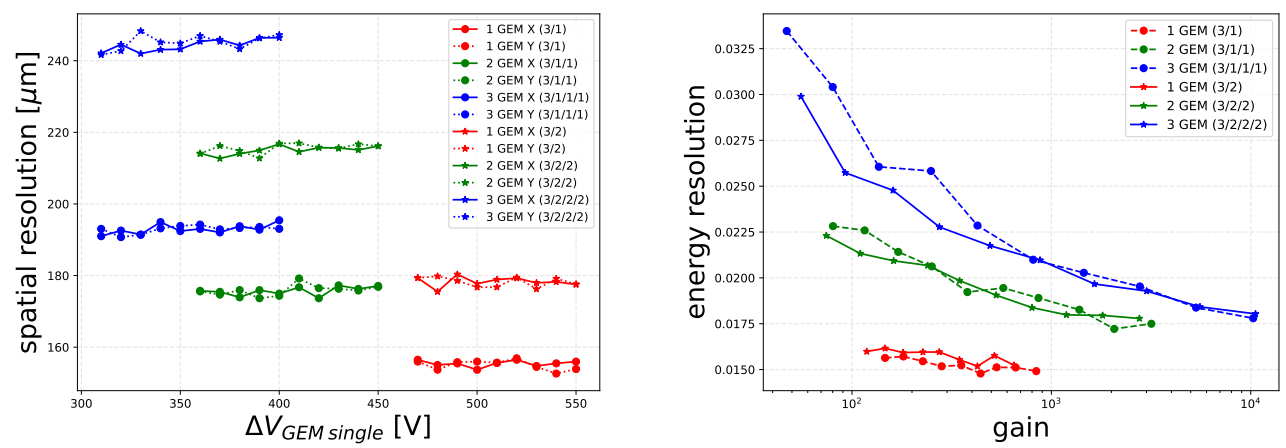

Figure 4: Left: Spatial resolution as a function of GEM voltage between top and bottom GEM electrodes for single (red), double (green), and triple (blue) GEMs. Right: Energy resolution as a function of gain for single (red), double (green), and triple (blue) GEMs.

\subsection{Spatial resolution}

The spatial resolution is one of the key parameters for tracking systems. It will largely influence the precision of a spectrometer and therefore the physics that can be accessed. It can be extracted from the width of the residual distribution reached on the anode plate. For a single GEM, the spatial resolution is about $150 \mu \mathrm{m}$. It can be seen that the distance from the first GEM to the readout plate increases by about $15 \mu \mathrm{m} / \mathrm{mm}$, as shown in the left panel of Fig 4. I.e. a triple GEM with a $2 \mathrm{~mm}$ transfer and induction gap has a spatial resolution of $240 \mu m(=150+15 \times 6)$.

\subsection{Energy resolution}

The energy resolution is central for GEM detectors working in proportional mode and other devices aiming for a measurement of the deposited energy. From the mean and the sigma of the gain distribution, the energy resolution has been estimated, as shown in the right panel of Fig. 4. As the layers of the GEM increase, the energy resolution deteriorates near gain 1000.

\subsection{Summary}

We have performed a comparative simulation study of single, double, and triple GEM systems with Garfield++ and ANSYS. As the number of GEM layers is increased, the gain increases by using a small delta GEM voltage. On the other hand, energy resolution deteriorates as the number of GEM layers is increased while maintaining the system at a constant gain. The spatial resolution becomes poorer as the distance between the first GEM and the anode increases. However, this difference is only about $15 \mu \mathrm{m} / \mathrm{mm}$. Lastly, while there are some differences in transparency and efficiency, single, double, and triple GEMs are pretty much the same.

\section{Acknowledgement}

We have been extremely fortunate to have the support and encouragement of many individuals as well as various organizations mostly by email and other discussions. We are thankful to Dr. 
Jeremie Alexandre Merlin, Dr. Rajendra Nath Patra, Dr. Rob Veenhof, Dr. Supratik Mukhopadhyay, Dr. Lia Lavezzi, Dr. Heinrich Schindler, Luis Felipe Ramirez Garcia, and the CERN service-desk. We would like to convey our gratitude to each and every one of them.

\section{References}

[1] G. Charpak, F. Sauli, Ann. Rev. Nucl. Part. Sci. 34, 285 (1984)

[2] A. Oed, Nucl. Instr. Meth., A262(1988),351

[3] F. Sauli, Nucl. Instr. Meth., A386(1997), 531

[4] Y. Giomataris, Nucl. Instr. Meth., A376(1996), 29

[5] M. Titov, arXiv: 1008.3736[physics.ins-dec], Gaseous Detectors: recent developments and applications (2010)

[6] S. Bachmann, et al., Nucl. Instrum. Meth. A 479, 294-308 (2002)

[7] Garfield++ CERN, https://garfieldpp.web.cern.ch/garfieldpp/

[8] I.B.Smirnov, Modeling of ionization produced by fast charged particles in gases, Nucl. Instrum. Meth.A, 554 (2005) 474

[9] S.F. Biagi, Monte Carlo simulation of electron drift and diffusion in counting gases under the influence of electric and magnetic fields, Nucl. Instrum. Meth. A 421 (1999) 234

[10] M. Titov, Nucl. Instrum. Meth., A 581, 25 (2007)

[11] R. Veenhof, “Garfield, recent developments”, NIM A 419 (2-3) (1998) 726

[12] CERN. Root data analysis framework, https://root.cern.ch

[13] O. Sahin et al., High-precision gas gain and energy transfer measurements in Ar-C O2 mixtures. Nucl. Ins. Meth. A 768, 104 (2014)

[14] W. Bonivento et al., A complete simulation of a triple-GEM detector, IEEE Trans.Nucl.Sci. 49,1638-1643 (2002) 